\title{
Liver Dysfunction and Oxidative Stress in Streptozotocin-Induced Diabetic Rats: Protective Role of Artemisia Turanica
}

\author{
Hassan Bgheri Yazdi ${ }^{1}$,Vida Hojati ${ }^{1}$, Abdolhossein Shiravi', Sara Hosseinian ${ }^{2,4}$, \\ Gholamhassan Vaezi ${ }^{1 *}$ Mousa-Al-Reza Hadjzadeh ${ }^{2,3 *}$ \\ ${ }^{1}$ Department of Biology, Damghan Branch, Islamic Azad University, Damghan, Iran \\ 2 Department of Physiology, School of Medicine, Mashhad University of Medical Sciences, Mashhad, Iran \\ ${ }^{3}$ Division of Neurocognitive Sciences, Psychiatry and Behavioral Sciences Research Center, Mashhad University of Medical Sciences, \\ Mashhad, Iran \\ ${ }^{4}$ Neurogenic Inflammation Research Center, Mashhad University of Medical Sciences, Mashhad, Iran
}

\section{Key Words}

diabetes mellitus, artemisia turanica, metformin, oxidative stress

\section{Abstract \\ Objectives: Oxidative stress plays a central role in di- abetes-induced complications. In the present study, the protevtive effect of Artemisia turanica (A. turanica) was evaluated against diabetes-induced liver oxidative stress and dysfunction.}

Methods: Fifty male Wistar rats were randomly divided into five groups: control, diabetic, diabetic + metformin, diabetic $+A$. turanica extract, and diabetic $+A$. turanica extract + metformin. Experimental diabetes was induced by a single-dose ( $55 \mathrm{mg} / \mathrm{kg}$, intraperitoneally (ip)) injection of streptozotocin (STZ). Metformin (300 $\mathrm{mg} / \mathrm{kg}$ ) and $A$. turanica extract $(70 \mathrm{mg} / \mathrm{kg}$ ) were orally administrated three days after STZ injection for four weeks. The levels of malondialdehyde (MDA), total thiol content and superoxide dismutase (SOD) and catalase activities were measured in the liver tissue. Serum glucose concentration, aspartate aminotransferase (AST)

\begin{tabular}{lll}
\hline Received: Aug 27, 2018 Reviewed: Mar 06, $2019 \quad$ Accepted: May 20, 2019 \\
\hline
\end{tabular}

$($ Chis is an Open-Access article distributed under the terms of the Creative Common Attribution Non-Commercial License (http://creativecommons.org/licenses/by-nc/4.0/) which permits unrestricted noncommercial use, distribution, and reproduction in any medium, provided the original work is properly cited.

( $₫$ This paper meets the requirements of KS X ISO 9706, ISO 9706-1994 and ANSI/NISO Z39.48-1992 (Permanence of Paper). and alanine aminotransferase (ALT) activities were also determined.

Results: In the diabetic group, serum glucose concentration, serum AST and ALT activities and liver MDA level were significantly higher while tissue total thiol content as well as catalase and SOD activities were lower, compared to the control group. Serum glucose in diabetic rats treated with metformin $+A$. turanica extract showed a significant decrease compared with the diabetic group. In all the $A$. turanica extract and metformin treated groups, serum ALT, tissue MDA level, total thiol content and SOD activity significantly improved compared with the diabetic rats. However, treatment of the diabetic rats only with metformin could not significantly change the activities of catalase and AST compared with the diabetic group.

Conclusion: These findings suggested that $A$. turanica extract had a therapeutic effect on liver dysfuncyion and oxidative stress induced by diabetes, that may be probably due to its antioxidant and antiinflammatory effects.

\section{Introduction}

Diabetes mellitus is a common health problem with

"Corresponding Author

Mousa-Al-Reza Hadjzadeh. Department of Physiology, Faculty of Medicine, Mashhad University of Medical Sciences (MUMS), University campus, Azadi Square, 9177948564, Mashhad, Iran.

Tel: +98-51-388-2856 Fax: +98-513-882-8564 E-mail: hajzadehmr@mums.ac.ir

Gholamhassan Vaezi. Department of Biology, Faculty of Basic Sciences, Islamic Azad University, Cheshme Ali Blvd, Damghan, Iran.

Tel: +98-367-163-9998

E-mail: gh.vaezi@yahoo.com

(C) 2019 Korean Pharmacopuncture Institute

http://www.journal.ac 
multiple etiology mainly characterized by hyperglycemia resulting from insulin secretion and/or function abnormalities [1]. Diabetes is also associated with major defects in lipid and protein metabolisms, thereby leading to many severe and life threatening complications including neuropathy, nephropathy, retinopathy, vasculopathy and hepatopathy [2]. Fibrosis, abnormal fat and glycogen deposition, cirrhosis and increased hepatic enzyme activities are some of liver abnormalities associated with diabetes [3]. Moreover, alterations in hepatic cell growth and cell number alter the liver size during diabetes. Increasing data suggests that free radicals generation resulted by glucose oxidation and protein glycation as well as defects in antioxidant defense systems play a critical role in pathogenesis of both types of diabetes $[4,5]$. It has been proposed that the most important cause of liver damage in diabetic patients is hyperglycemia-induced oxidative stress and subsequent disturbance in carbohydrate, protein and lipid metabolisms [6]. These events, in turn, lead to further oxidative stress and activation of inflammatory cascades [6]. Nowadays, reduction of hyperglycemia using edible drugs and injection of insulin are the most common strategies for treatment of diabetes. However, gradual resistance to these agents, in addition to their various adverse effects, has increased the need for finding alternative therapies with less or even no side effects for diabetic patients. In recent years, the use of herbal medications has developed to minimize hyperglycemia and other diabetes associated complications. In various investigations, the protective action of herbal antioxidants has been reported in scavenging free radicals and improving liver dysfunction in diabetic experimental models [7-9].

Genus Artemisia is one of the most important and largest genera in the family Asteraceae (Compositae). This genus comprises about 500 species, most of which are medicinal plants and have been traditionally used all over the world [10]. Artemisia turanica (A. turanica) commonly known as "Dermane Ghermez" grows mainly in the northeast of Iran [11]. There are different compounds in the essential oil of $A$. turanica including 1,8-cineol, chrysanthenone, davanone, cis-verbenyl, camphor and oxygen containing monoterpenoids [12]. It has been reported that $A$. turanica possesses different biological activities including antimalarial, anti-fungal, cytotoxic and apoptotic properties $[13,14]$. The current study was undertaken to determine whether Artemisia turanica aqueous-ethanolic extract could improve the liver dysfunction and oxidative stress in STZ-induced diabetic rats.

\section{Materials and Methods}

\subsection{Extract preparation}

Aerial parts of the plant were collected from Mazrae Sheikh, a village in Khaf City, (Razavi Khorasan Province, Iran) and were identified by a botanist in the Herbarium of School of Pharmacy, the Mashhad University of Medical Sciences, Mashhad, Iran (specimen number 12572). The plant was washed, dried, powdered and macerated with $70 \%$ ethanol with occasional shaking and stirring.
Then, the filtered mixture was kept at $45^{\circ} \mathrm{C}$ for ethanol evaporation, yielding a blackish-brown concentrate. The prepared extract was kept at $4^{\circ} \mathrm{C}$ prior to use.

\subsection{Drugs and kits}

Metformin (Met) powder was purchased from Samisaz Company (Mashhad, Iran). STZ was obtained from Sigma-Aldrich (St Louis, MO, USA). Moreover, Glucose, aspartate aminotransferase (AST) and alanine aminotransferase (ALT) kits were provided from Pars Azmoon Company (Tehran, Iran).

\subsection{Animals}

Fifty male Wistar rats ( 12 weeks old, weighed $200 \pm 20$ g) were obtained from the Animal House of the School of Medicine, the Mashhad University of Medical Sciences, Mashhad, Iran. All the animals were maintained at $22 \pm$ $2{ }^{\circ} \mathrm{C}$ and $12 \mathrm{~h}$ light/dark cycle. All the experiments were performed under the authority of the Mashhad University of Medical Sciences and the norms of international animal ethics were followed.

\subsection{Experimental Design}

The animals were randomly divided into five groups $(\mathrm{n}=$ 10) as follows:

Control: free access to food and water with an intraperitoneal (ip) injection of saline at the first of the study

Diabetic: single dose ip injection of STZ ( $55 \mathrm{mg} / \mathrm{kg}$ ) for induction of diabetes

Diabetic + Met: oral administration (by gavage) of metformin $(300 \mathrm{mg} / \mathrm{kg}$ ) three days after STZ injection for four consecutive weeks

Diabetic + Ext: oral administration (by gavage) of Artemisia turanica extract $(70 \mathrm{mg} / \mathrm{kg})$ three days after STZ injection for four consecutive weeks.

Diabetic + Ext + Met: oral administration (by gavage) of Artemisia turanica extract $(70 \mathrm{mg} / \mathrm{kg})$ and metformin (300 $\mathrm{mg} / \mathrm{kg}$ ) three days after STZ injection for four consecutive weeks.

At the end of the experiment, serum samples were collected from the orbital sinus and kept at $-20^{\circ} \mathrm{C}$ for serum glucose and hepatic enzymes assessment. Then, all the animals were humanely killed and the liver tissue was quickly removed and conserved for oxidative stress assessment.

\subsection{Assessment of serum biochemical parameters}

Serum glucose concentration as well as AST and ALT activities were detected by the commercial kits based on the manufacturer's instructions.

\subsection{Assessment of oxidative stress mark- ers}




\subsubsection{Malondialdehyde (MDA) measure- ment}

MDA as an index of lipid peroxidation reacts with thiobarbituric acid (TBA) as a thiobarbituric acid reactive substance to produce a red colored complex. It has a peak absorbance at $535 \mathrm{~nm}$. In this stuyd, $15 \mathrm{~g}$ TCA, $0.375 \mathrm{~g}$ TBA and $2 \mathrm{ml} \mathrm{HCl}$ were mixed, $2 \mathrm{ml}$ of this mixture was added to $1 \mathrm{ml}$ of serum or kidney homogenate in a centrifuge tube, and the mixture was heated for 50 min in a boiling water bath. After cooling, the mixture centrifuged at 1000 rpm for $10 \mathrm{~min}$. The absorbance (A) of the colored layer was read at $535 \mathrm{~nm}$. MDA concentration was calculated from the following equation [15].

$$
\mathrm{C}(\mathrm{M})=\mathrm{A} / 1.56 \times 105
$$

\subsubsection{Total thiol content measurement}

Total-SH groups were measured using DTNB as the reagent. The reagent reacted with the $\mathrm{SH}$ groups to produce a yellow colored complex which had a peak absorbance at $412 \mathrm{~nm}$. Afterwards, $1 \mathrm{ml}$ Tris-EDTA buffer was added to $50 \mu \mathrm{l}$ of serum or kidney homogenate and absorbance was read at $412 \mathrm{~nm}$ against Tris-EDTA buffer alone (A1). Then, $20 \mu \mathrm{l}$ DTNB reagents ( $10 \mathrm{mM}$ in methanol) were added to the mixture and after $10 \mathrm{~min}$, the sample absorbance was read again (A2). The absorbance of the DTNB reagents was also read as a blank (B). Total thiol concentration $(\mathrm{mM})$ was calculated from the following equation [16]:

Total thiol concentration $(\mathrm{mM})=(\mathrm{A} 2-\mathrm{A} 1-\mathrm{B}) \times 1.07 / 0.05$ $\times 13.6$

\subsubsection{Assessment of antioxidant enzymes activities}

Superoxide dismutase (SOD) activity was determined in the stomach tissue by the method of Madesh [17]. In a colorimetric assay, the SOD activity was measured at $570 \mathrm{~nm}$. One unit of SOD was defined as the amount of enzyme required to inhibit the rate of MTT reduction by $50 \%$. The results were shown as unit per milligram protein. Catalase activity was measured according to the method of Aebi with hydrogen peroxide $(30 \mathrm{mM})$ as the substrate. One unit of catalase activity was determined as the micromoles of the hydrogen peroxide consumed per milligram of protein sample.

\subsection{Statistical analysis}

The data were expressed as mean \pm SEM. One-way ANOVA was run followed by a post hoc LSD comparison test. Statistical significance was considered as $\mathrm{p}<0.05$.

\section{Result}

\subsection{The effect of $A$. turanica extract and metformin on serum biochemical parame- ters}

Serum glucose concentration in the diabetic group significantly increased compared with the control group ( $\mathrm{p}<$ 0.001). However, treatment of diabetic rats with metformin and $A$. turanica extract separately caused a significant decrease in blood glucose compared to the diabetic rats ( $\mathrm{p}<0.01 \& \mathrm{p}<0.001$, respectively) (Fig. 1). As shown in Table 1, serum AST and ALT activities showed significant increase in the diabetic group compared to those of the control group $(\mathrm{p}<0.05)$ (Table 1$)$. In the diabetic + Ext and diabetic + Ext + Met groups, serum AST activity was significantly lower compared to the diabetic group $(\mathrm{p}<0.05)$ (Table 1). Interestingly, compared with the control group, serum ALT activity significantly decreased ( $p<0.001)$ in the diabtic + Ext and diabtic + Ext + Met groups, but showed no significant change in the diabetic + Met group. Serum ALT activity in all the $A$. turanica extract and metformin treated groups significantly decreased compared with the diabetic rats $(\mathrm{p}<0.05, \mathrm{p}<0.01 \& \mathrm{p}<0.001$ respectively) (Table 1).

\subsection{The effect of $A$. turanica extract and metformin on hepatic oxidative stress bio- markers liver MDA concentration}

MDA concentration was significantly higher in the diabetic rats than in the control animals $(\mathrm{p}<0.001)$ (Fig. 2). However, the separate and concomitant treatment of the diabetic rats with $A$. turanica extract and metformin significantly decreased the liver tissue level of MDA as compared with the diabetic group ( $\mathrm{p}<0.001)$ (Fig. 2). In these groups, MDA concentration showed no significant change when compared with the control group.

Table 1 The effect of A. turanica on serum AST and ALT activities in all experimental groups.

\begin{tabular}{|c|c|c|c|c|c|}
\hline & Control & Diabetic & Diabetic + Met & Diabetic + Ext & Diabetic + Ext + Met \\
\hline $\operatorname{AST}(\mathrm{U} / \mathrm{L})$ & $179.6 \pm 20.99$ & $365.8 \pm 30.4^{*}$ & $313.5 \pm 98.79$ & $221.16 \pm 81.77^{\#}$ & $217.5 \pm 54.69^{\#}$ \\
\hline $\operatorname{ALT}(\mathrm{U} / \mathrm{L})$ & $101 \pm 20.98$ & $201.8 \pm 47.05^{*}$ & $103.8 \pm 40.2^{\#}$ & $37.8 \pm 15.49^{* * * \# \# \#}$ & $31 \pm 8.5^{* * * \# \# \#}$ \\
\hline
\end{tabular}

Met: metformin, Ext: Artemisia turanica extract, AST: aspartate aminotransferase, ALT: alanine aminotransferase.

All data were expressed as mean $\pm \operatorname{SEM}(n=10$ in each group).

${ }^{*} \mathrm{p}<0.05,{ }^{* * *} \mathrm{p}<0.001$ compared to control group.

${ }^{\#} \mathrm{p}<0.05,{ }^{\# \#} \mathrm{p}<0.001$ compared to diabetic group. 


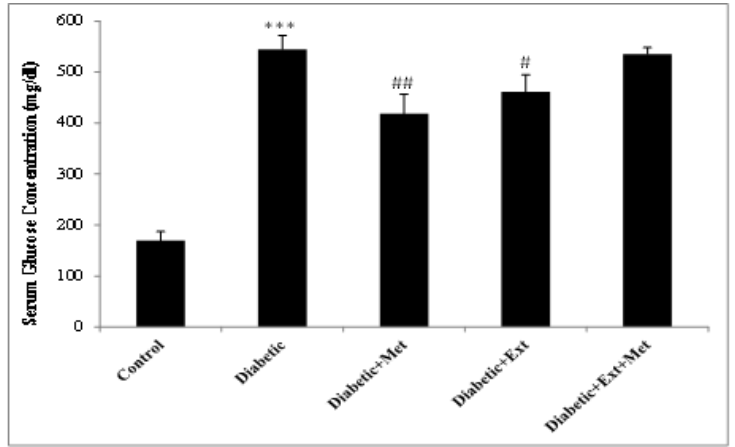

Figure 1 Glucose concentration in all experimental groups. Values are the Mean \pm SEM. The data were analyzed using one-way ANOVA \& post hoc LSD.

*** $\mathrm{p}<0.001$ compared to control group.

\# $\mathrm{p}<0.05$ and \#\# $\mathrm{p}<0.01$ compared to diabetic group

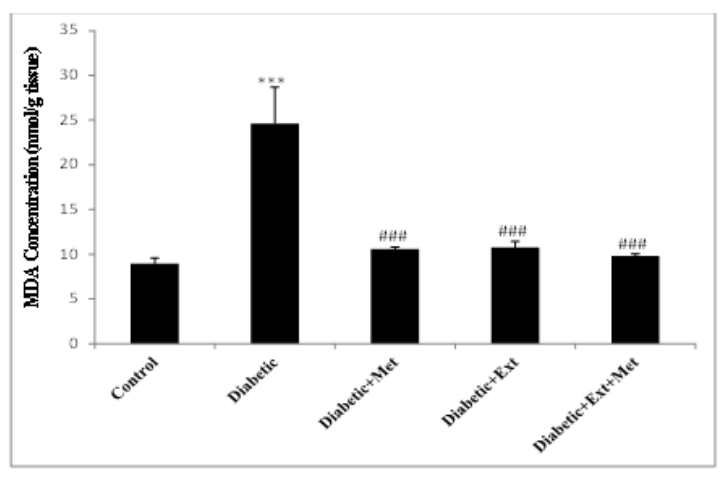

Figure 2 Liver MDA concentration in all experimental groups. Values are the Mean \pm SEM. The data were analyzed using oneway ANOVA \& post hoc LSD.

*** $\mathrm{p}<0.001$ compared to control group.

\#\#\# $\mathrm{p}<0.001$ compared to diabetic group.

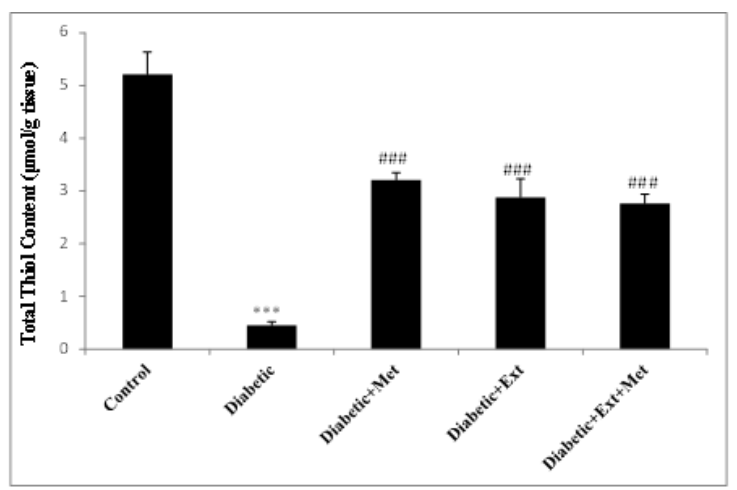

Figure 3 Liver total thiol concentration in all experimental groups. Values are the Mean \pm SEM. The data were analyzed using one-way ANOVA \& post hoc LSD.

*** $\mathrm{p}<0.001$ compared to control group \#\#\# $\mathrm{p}<0.01$ compared to diabetic group

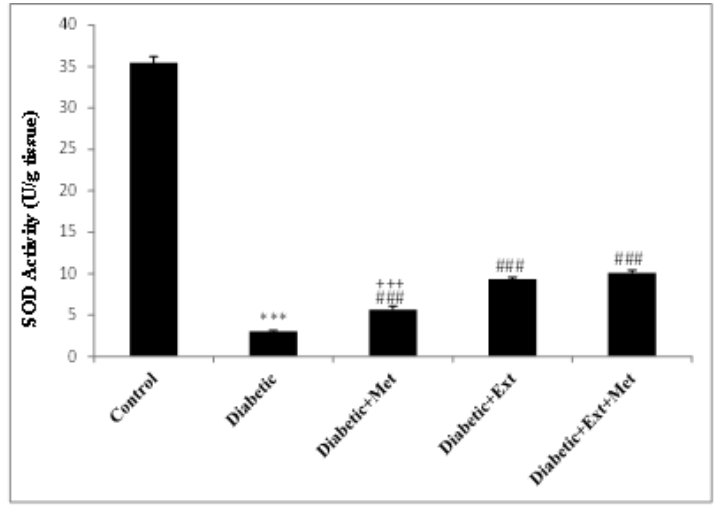

Figure 4 Liver superoxide dismutase (SOD) activity in all experimental groups. Values are the Mean \pm SEM. The data were analyzed using one-way ANOVA \& post hoc LSD.

*** $\mathrm{p}<0.001$ compared to control group.

\#\#\# $\mathrm{p}<0.001$ compared to diabetic group.

$+++\mathrm{p}<0.001$ compared to Diabetic+Ext+Met group.

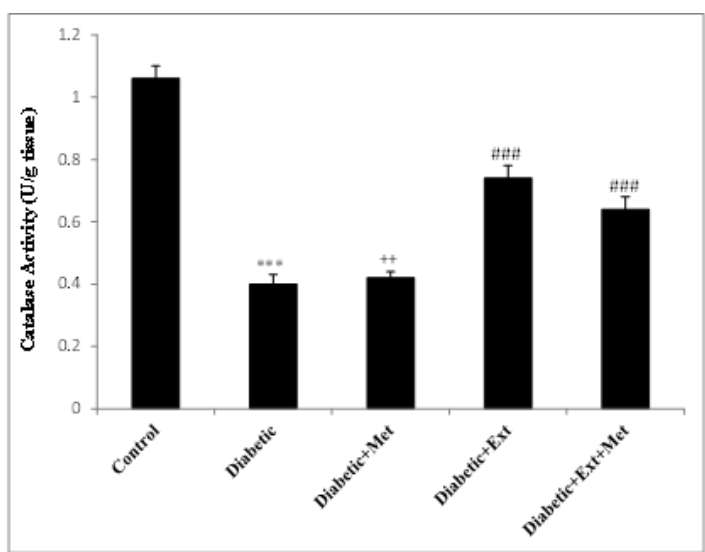

Figure 5 Liver catalase activity in all experimental groups. Values are the Mean \pm SEM. The data were analyzed using one-way ANOVA \& post hoc LSD.

*** $\mathrm{p}<0.001$ compared to control group.

\#\#\# $\mathrm{p}<0.001$ compared to diabetic group.

$++\mathrm{p}<0.01$ compared to Diabetic+Ext+Met group.

\subsection{Liver total thiol content}

As shown in Fig. 3, total thiol content in the diabetic rats was significantly lower than control animals $(\mathrm{p}<0.001)$ (Fig. 3). In all the metformin and $A$. turanica extract treated groups, total thiol content showed a significant increase compared with the diabetic group $(\mathrm{p}<0.001)$ (Fig. 3$)$.

\subsection{Liver antioxidant enzymes activities}

Figures 4 and 5 demonstrate the activities of SOD and catalase enzymes in the liver tissue in all the experimental groups. The results showed that SOD and catalase activities significantly decreased in the diabetic group compared to the control group $(\mathrm{p}<0.001)$. Separate and 
concomitant treatment of the diabetic rats with $A$. turanica extract and metformin significantly increased the SOD activity as compared with the diabetic group $(\mathrm{p}<0.001)$ (Fig. 4). However, catalase activity showed a significant increase in the diabetic + Ext and diabetic + Ext + Met groups $(\mathrm{p}<0.001)$ (Fig. 5). Compared to the diabetic + Ext + Met group, SOD and catalase activities were significantly lower than those of the diabetic + Ext and diabetic + Met groups $(\mathrm{p}<0.001 \& \mathrm{p}<0.01$, respectively) (Figs. $4 \& 5)$.

\section{Discussion}

In the current study, administration of $A$. turanica extract separately or concomitant with metformin significantly improved serum alterations in hepatic enzymes and liver tissue oxidative damage induced by diabetes. In the present investigation, in agreement with previous studies, we showed that STZ was significantly able to increase serum glucose concentration, which confirmed the accuracy of the present study in induction of diabetes. STZ-induced diabetes was also associated with significant alterations in liver dysfunction and oxidative stress markers. Hyperglycemia is the most common feature in both types of diabetes. Previous studies suggest that free radicals generation and simultaneous defect in cells antioxidant capacity are the main mechanisms for diabetes complications [18]. Different endogenous organs are highly affected by diabetes with liver being one of the most important organs [19]. It has been reported that hyperglycemia-induced oxidative stress and subsequent disturbance in carbohydrate, protein and lipid metabolisms are the most important causes of liver damage in diabetic patients [6]. Based on our results, treatment of the STZ-induced diabetic rats separately with $A$. turanica extract and metformin significantly decreased blood glucose level as compared to the untreated diabetic rats. In the same context, Ghazanfar et al. (2014) reported significant decrease in blood level of glucose in STZ-induced diabetic animals receiving Artemisia amygdalina extract [20]. The antidiabetic activities of Artemisia judaica, Artemisia annua and Artemisia herba-alba have also been reported [21-23]. The hypoglycemic effect of $A$. turanica extract in our study might be due to stimulation of insulin secretion from $§$ cells and reduction of tissue resistance to insulin, mainly by its terpenoid compounds [24, 25].

However, the exact mechanism for the lack of hypoglycemic action of metformin and $A$. turanica co-administration in the diabetic rats is not clear; however, these two factors appear to counteract each other in a way that is not elucidated. In the current study, in all the metformin and A. turanica extract treated groups, MDA concentration significantly decreased but the content of the total thiol groups and SOD activity significantly increased compared with the diabetic untreated rats. However, treatment of the diabetic rats with metformin separately could not change the catalase activity when compared with the diabetic group. These results are in agreement with previous studies describing the protective role of different species of Artemisia on liver oxidative damage induced by diabetes [26-28]. Therefore, the beneficial action of $A$. turanica on liver tissue oxidative damage in the STZ-induced di- abetic rats might be due to its antioxidant effects mainly by terpenoid compounds [29]. As observed in our results, treatment of the diabetic animals only with $A$. turanica led to more protection, approximately $25 \%$, against antioxidant enzymes dysfunction, as compared to the co-administration of metformin and $A$. turanica extract. In the present work, diabetes was also associated with a significant increase in serum ALT and AST activities. AST and ALT are two well-known hepatic enzymes that cause liver cell damage in case of having elevated serum activities, as observed in the diabetic rats. We observed that oral administration of $A$. turanica extract alone and in combination with metformin significantly attenuated the elevated activities of AST and ALT.

This may be due to the protective action of the extract possibly via its antioxidant effect in reversing liver damage induced by diabetes. Similar findings were also reported by Taofik et al. (2013) using Artemisia afra aqueous extract in diabetic rats [30].

\section{Conclusion}

In conclusion, the findings of the present study revealed that $A$. turanica extract could improve the liver oxidative damage and dysfunction in the STZ-treated diabetic rats. However, our results demonstrated that separate administration of $A$. turanica exerted more protection against liver injury iduced by diabetes than the co-adminstration of metformin and $A$. turanica. Further investigations are required to elucidate mechanisms for beneficial actions of melatonin during diabetes.

\section{Conflict of interest}

The authors declare that there is no conflict of financial interest.

\section{References}

1. Ruch W, Zumsteg U. Diabetes or hyperglycemia? Schweizerische medizinische Wochenschrift. 1988;118(8):264-266.

2. Amos AF, McCarty DJ, Zimmet P. The rising global burden of diabetes and its complications: estimates and projections to the year 2010. Diabetic medicine. $1997 ; 14(\mathrm{~S} 5)$.

3. Levinthal GN, Tavill AS. Liver disease and diabetes mellitus. Clinical diabetes. 1999;17(2):73.

4. Ahmad FK, He Z, King GL. Molecular targets of diabetic cardiovascular complications. Current drug targets. 2005;6(4):487-494.

5. Jiang Z-Y, Woollard A, Wolff SP. Hydrogen peroxide production during experimental protein glycation. Febs Letters. 1990;268(1):69-71.

6. Mohamed J, Nafizah AN, Zariyantey A, Budin SB. Mechanisms of Diabetes-Induced Liver Damage: The role of oxidative stress and inflammation. Sultan Qaboos University Medical Journal. 2016;16(2):e132.

7. Balasubramanian T, Senthilkumar G, Karthikeyan M, Chatterjee TK. Protective effect of ethyl acetate fraction 
of stereospermum suaveolens against hepatic oxidative stress in STZ diabetic rats. Journal of traditional and complementary medicine. 2013;3(3):175-181.

8. Hassani FV, Mehri S, Abnous K, Birner-Gruenberger R, Hosseinzadeh $\mathrm{H}$. Protective effect of crocin on BPA-induced liver toxicity in rats through inhibition of oxidative stress and downregulation of MAPK and MAPKAP signaling pathway and miRNA-122 expression. Food and Chemical Toxicology. 2017;107:395-405.

9. Alam MM, Meerza D, Naseem I. Protective effect of quercetin on hyperglycemia, oxidative stress and DNA damage in alloxan induced type 2 diabetic mice. Life sciences. 2014;109(1):8-14.

10. Bora KS, Sharma A. The genus Artemisia: a comprehensive review. Pharmaceutical Biology. 2011;49(1):101109.

11. V M. A Dictionary of Iranian Plant Names. Tehran: Farhang Moaser; 1998.

12. Khayyat $\mathrm{MH}$, Karimi H. Composition of the volatile oils of three different species of Artemisia. Iranian Journal of Pharmaceutical Sciences. 2005;1(1):33-37.

13. Behravan J, Ramezani M, Hassanzadeh M, Eliaspour N, Sabeti Z. Cytotoxic and Antimycotic Activities of essential oil of Artemisia turanica Krasch from Iran. Journal of Essential Oil Bearing Plants. 2006;9(2):196-203.

14. Taherkhani M, Rustaiyan A, Nahrevanian H, Naeimi S, Taherkhani T. Comparison of antimalarial activity of Artemisia turanica extract with current drugs in vivo. Journal of vector borne diseases. 2013;50(1):51.

15. Khodabandehloo F, Hosseini M, Rajaei Z, Soukhtanloo M, Farrokhi E, Rezaeipour M. Brain tissue oxidative damage as a possible mechanism for the deleterious effect of a chronic high dose of estradiol on learning and memory in ovariectomized rats. Arquivos de Neuro-psiquiatria. 2013;71(5):313-319.

16. Ellman GL. Tissue sulfhydryl groups. Archives of biochemistry and biophysics. 1959;82(1):70-77.

17. Madesh M, Balasubramanian KA. Microtiter plate assay for superoxide dismutase using MTT reduction by superoxide. Indian J Biochem Biophys. 1998;35(3):184188. PubMed PMID: 9803669. Epub 1998/11/06. eng.

18. Giacco F, Brownlee M. Oxidative stress and diabetic complications. Circulation research. 2010;107(9):10581070.

19. Ahmadieh H, Azar ST. Liver disease and diabetes: association, pathophysiology, and management. Diabetes research and clinical practice. 2014;104(1):53-62.

20. Ghazanfar K, Ganai BA, Akbar S, Mubashir K, Dar SA, Dar MY, et al. Antidiabetic activity of Artemisia amygdalina Decne in streptozotocin induced diabetic rats. BioMed research international. 2014;2014:Article number 185676.

21. Helal EG, Aouf NA, Khattab AM, Zoair MA. ANTI-DIABETIC EFFECT OF ARTEMISIA ANNUA (KAYSOM) IN ALLOXAN-INDUCED DIABETIC RATS. The Egyptian Journal of Hospital Medicine. 2014;57:422-430.

22. Al-Waili NSD. TREATMENT OF DIABETES MELLITUS BY ARTEMISIA HERBA-ALBA EXTRACT: PRELIMINARY STUDY. Clinical and Experimental Pharmacology and Physiology. 1986;13(7):569-574.

23. Nofal SM, Mahmoud SS, Ramadan A, Soliman G, Fawzy
R. Anti-diabetic effect of Artemisia judaica extracts. Research Journal of Medicine and Medical Sciences. 2009;4(1):42-48.

24. Nazaruk J, Borzym-Kluczyk M. The role of triterpenes in the management of diabetes mellitus and its complications. Phytochemistry Reviews. 2015;14(4):675-690.

25. Stainsloss I, Sankarann M. Chemotherapeutic effect of 3, 3'-Diindolylmethane encapsulated chitosan nanoparticles on 7, 12-Dimethylbenz (a) anthracene induced mammary cancer - A dose dependent study. New Horizons in Translational Medicine. 2016;3(1):1-8

26. Han K-H, Jeon Y-J, Athukorala Y, Choi K-D, Kim C-J, Cho J-K, et al. A water extract of Artemisia capillaris prevents 2, 2'-azobis (2-amidinopropane) dihydrochloride-induced liver damage in rats. Journal of medicinal food. 2006;9(3):342-347.

27. Kim MH, Seo JY, Liu KH, Kim J-S. Protective effect of Artemisia annua L. extract against galactose-induced oxidative stress in mice. PloS one. 2014;9(7):e101486.

28. Sefi M, Bouaziz H, Soudani N, Boudawara T, Zeghal N. Fenthion induced-oxidative stress in the liver of adult rats and their progeny: Alleviation by Artemisia campestris. Pesticide biochemistry and physiology. 2011;101(2):71-79.

29. Grassmann J. Terpenoids as plant antioxidants. Vitamins \& Hormones. 2005;72:505-535.

30. Sunmonu TO, Afolayan AJ. Evaluation of antidiabetic activity and associated toxicity of Artemisia afra aqueous extract in wistar rats. Evidence-Based Complementary and Alternative Medicine. 2013;2013:Article number 929074 . 